\title{
A multicenter, prospective, randomized clinical trial comparing tension-free vaginal tape surgery and no treatment for the management of stress urinary incontinence in elderly women
}

Campeau L, Tu LM, Lemieux MC, Naud A, Karsenty G, Schick E, Corcos J

Department of Urology, Sir Mortimer B. Davis-Jewish General Hospital, McGill University, Montreal, Canada

Neurourol Urodyn. 2007; 26: 990-4

Aims: The aim of our study was to test the hypothesis that elderly women undergoing tension-free vaginal tape surgery (TVT) will have a better quality of life (QOL) and satisfaction compared to non-treated women despite age- and technique-related potential morbidity.

Methods: This multicenter, prospective, randomized, controlled trial enrolled a total of 69 women aged over 70 years who initially consented to be randomized to either undergo immediate TVT surgery or to wait for 6 months before submitting to the same surgery (control group). The main outcomes measured at every visit (pre-randomization, 8-12 weeks and 6 months) consisted of the Incontinence-Quality of Life (I-QOL) Questionnaire, the Patient Satisfaction Questionnaire and the Urinary Problems Self-assessment Questionnaire, among others.

Results: The analysis included 31 patients in the immediate surgery group and 27 subjects in the control group. Peri-operative complications in the immediate surgery group were bladder perforation $(22.6 \%)$, urinary retention $(12.9 \%)$, urinary tract infection $(3.2 \%)$ and de novo urgency (3.2\%). At 6 months, the mean I-QOL scores for the TVT and control groups were respectively $96.5+/-15.5$ and 61.6 +/- $19.8(\mathrm{P}<0.0001)$; mean Patient Satisfaction scores were respectively $8.0+/-2.7$ and $2.0+/-2.4(\mathrm{P}<0.0001)$; and mean Urinary Problems scores were respectively $4.5+/-4.3$ and $11.6+/-3.5(\mathrm{P}<0.0001)$.

Conclusion: At 6 months post-randomization, the group of elderly women who underwent immediate TVT surgery showed a significant improvement in QOL, patient satisfaction and less urinary problems compared to the group of women waiting for the same surgery

\section{Editorial Comment}

The authors provide a study with the primary goal of deciding whether elderly women who underwent surgery with its attendant risks would have an improved quality of life and satisfaction than those patients who deferred surgery and merely continued on in their state of incontinence or in simpler terms: should an elderly woman have surgery or just live with her symptoms. The population studied included patients that either refused or failed conservative therapy and were on no medication for bladder dysfunction (including overactive bladder or urinary tract infection). The patients had no evidence of detrusor overactivity and had normal compliance on cystometrogram. The patient population was randomized to either having surgery immediately or waiting six months and being reevaluated and then having their surgery at that time. The metrics for both groups were quantified at baseline and again at 6 months and then compared. The tools that were utilized to assess the patient's quality of life included the IQOL Questionnaire; Patient Satisfaction scores; in addition to a Urinary Problems quantification. The authors found that patients who had the surgery had improvement in the measured parameters over those practicing watchful waiting thus supporting their hypothesis that surgery was worthwhile over watchful waiting in the elderly population.

This is an excellent paper to review when pondering whether to offer surgery to an elderly woman versus telling her to live with her problem. Many times, the surgeon must make the simple but really complex assessment of whether the treatment will outweigh the cure; this report emboldens one to choose intervention. 
The study begs the authors to take the next step of determining if the same promise of therapy can be applied to the super elderly population such as those of 80,85 or 90 years of age.

\author{
Dr. Steven P. Petrou \\ Professor of Urology, Associate Dean \\ Mayo School of Graduate Medical Education \\ Jacksonville, Florida, USA \\ E-mail: petrou.steven@mayo.edu
}

\title{
PEDIATRIC UROLOGY
}

doi: $10.1590 / S 1677-553820100002000030$

\section{Improvement in vesicoureteral reflux grade on serial imaging predicts resolution}

Cannon GM Jr, Arahna AA, Graham DA, Passerotti CC, Silva A, Retik AB, Nguyen HT

Children's Hospital Boston, Harvard Medical School, Boston, Massachusetts, USA

J Urol. 2010; 183: 709-13

Purpose: When children are initially diagnosed with vesicoureteral reflux most undergo a period of antibiotic prophylaxis followed by serial imaging. Although improvement in reflux grade through time presumably predicts eventual resolution, the significance of changing grade through time is unknown. We examined whether improvement in reflux on serial imaging predicts resolution.

Materials and Methods: We retrospectively reviewed 1,761 children diagnosed with vesicoureteral reflux, of which 965 had a minimum of 2 years of follow-up. We examined initial reflux grade and grade on serial imaging up to 5 years after the original diagnosis. For each child it was determined whether reflux was resolved, eventually resolved or never resolved. Groups were further stratified by clinical characteristics.

Results: Multivariate analysis revealed that male gender ( $\mathrm{HR} 1.33, \mathrm{p}=0.05)$, age younger than 1 year at diagnosis (HR 1.35, p = 0.004), lower grade at presentation (grade I HR 2.2, grade II HR 1.96, grade III HR 1.33; $\mathrm{p}<0.001$ ) and unilateral reflux (HR 1.39, $\mathrm{p}=0.001$ ) were all independent predictors of reflux resolution. Multivariate analysis also showed that reflux improvement on imaging 1 year after diagnosis (HR 3.14, p $<0.0001$ ) and improvement from the previous year at any point during follow-up (HR 1.8, $\mathrm{p}=0.009$ ) were independent predictors of reflux resolution.

Conclusions: Consistent with previous findings, male gender, lower reflux grade at presentation, age less than 1 year at presentation and unilateral reflux were all predictive of reflux resolution. Our analysis also demonstrated that improvement in reflux grade on imaging study 1 year after diagnosis was predictive of resolution, and that reflux improvement from the previous year at any point during follow-up was an independent predictor of resolution. This information will prove valuable in clinical counseling and therapeutic decision making.

\section{Editorial Comment}

The authors utilized their database of over 1,700 children with vesicoureteral reflux to determine whether improvement in reflux grade on serial imaging would predict resolution of reflux. They were able to identify 965 patients who had 2-5 years of follow-up for the study and then performed a multivariate analysis to identify predictors of resolution. Nuclear cystograms were performed routinely for follow-up studies in these children, therefore they considered Grade I reflux on a nuclear cystogram to be equivalent to a grade 1 on VCUG study. A nuclear cystogram with Grade II reflux was equivalent to a VCUG with Grade II and III on VCUG and Grade III reflux on nuclear cystogram was equivalent to Grade IV and V reflux on VCUG. Their 